\section{Computación Evolutiva y Teoría de Juegos: Un híbrido para la automatización en sistemas de soporte a la negociación}

\author{
Recepción: Julio de 2006 / Aceptación: Octubre 2006
}

(1) Oswaldo Velez-Langs

(2) Henry Gómez Rocha

(3) John Acosta Parejo

\section{RESUMEN}

Este articulo trata sobre los métodos de análisis de decisión y de apoyo a la decisión de grupos, desplegados sobre la web, con miras a involucrar al público en las decisiones que les afectan, es así como vemos en internet una oportunidad de acortar la brecha entre regidores y regidos. Podemos resumir el objeto principal que persigue este trabajo como proponer e implementar un esquema de negociación automatizada sobre la web, el cual esté basado en la computación evolutiva y la teoría de juegos.

Palabras Clave: Negociación automatizada, sistemas de soporte a la decisión, teoría de juegos, computación evolutiva.

Evolutive COMPUTING AND GAMES THEORY: A HIBRID FOR THE AUTOMATIZA TION IN BUSINESS SUPPORT SYSTEMS

ABSTRACT

This paper is about the decisión analysis methods for group decisions over the web, in reason of involve the people in the different decisions that interest them, we have in internet one chance for to reduce the gap between governor and governed We can summarize the main object that pursues this like to propose one automated negotiation approach in the web, which be based on the evolutionary computation and the game theory.

Key words: Automated negociation, decision support systems, game theory, evolutionary computation.
I N T R O D U C C I Ó N

Los problemas de negociación son por excelencia no-algorítmicos y las soluciones a este tipo de problemas se obtienen luego de un proceso dispendioso y por ende sus resultados son difíciles de evaluar, es así como el modelamiento y soporte al problema de la negociación propone un reto muy interesante a los campos de Ciencia de la Decisión, Investigación de Operaciones e Inteligencia Artificial.

La propuesta presentada aquí se enmarca dentro de la denominada: Democracia Electrónica [14] (ver Figura 1), en la cual se pretende implantar un proceso democrático participativo que persigue un acuerdo entre grupos de personas con conflicto de intereses en asuntos concernientes a la sociedad, lo cual hace necesario plantear modelos de negociación bajo los cuales se interactúe para encontrar la solución más óptima, el soporte que se da a dicho proceso esta habilitado por internet.

En nuestros tiempos de digitalización no es sorprendente escuchar hablar de términos como gobierno electrónico (e-government), administración electrónica (e-administration) y democracia electrónica (edemocracia), en cada uno de estos se discute y promueve la participación pública en procesos de decisión que afectan a diversos interesados de este mismo público.

Posteriormente a la experiencias de Portoalegre, hay por ejemplo en Europa diversas municipalidades que permiten a sus ciudadanos participar (parcialmente) en la ejecución de los presupuestos municipales, a estos también se les denomina presupuestos participativos. En palabras de Souza: "Los Presupuestos Participativos constituyen una proposición universal, directa y voluntaria de una clase de democracia que da a la población la oportunidad de discutir acerca del presupuesto y temas de interés público, tomando decisiones relevantes. Los ciudadanos no sólo votan, sino también logran involucrarse" [22].

Entre los muchos potenciales beneficios de los presupuestos participativos, podemos destacar [23]:

- Legitimación

- Acerca las decisiones a los ciudadanos

- El público toma las decisiones en público

- Mitigación de alienación y apatía

- Transparencia

(1) Profesor del Departamento de Informática, Estadística y Telemática, Universidad Rey Juan Carlos (España) E-mail: oswaldo.velez.langs@urjc.es

(2) Universidad del Magdalena (Colombia) E-mail: hagr500@yahoo.com.mx

(3) Universidad del Magdalena (Colombia) E-mail: jhon_acosta386@yahoo.com.mx 


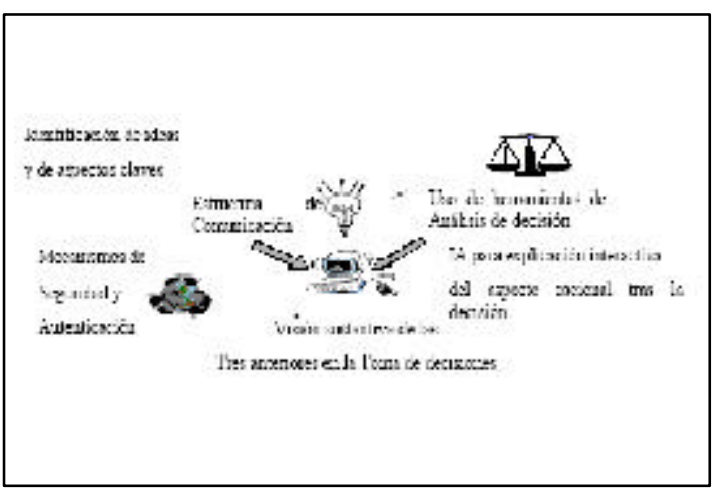

Figura 1. Esquema general de la Democracia Electrónica (Adaptado de [13])

Nuestra intención procura utilizar métodos de análisis de decisión y de apoyo a la decisión de grupos, desplegado sobre la web, con miras a involucrar al público en las decisiones que les afecta. Es así, como vemos en internet una oportunidad de acortar la brecha entre quienes rigen y quienes son regidos.

El problema surge debido a que dichos modelos por lo general se basan en negociaciones multi-criterio, es decir, con múltiples variables y por tal razón se recae en un proceso complejo, generando un espacio de búsqueda de la solución mas óptima extenso, demandando mucho tiempo e incluso convirtiéndose en un proceso demasiado tedioso. Otro problema que conllevan estas dinámicas es la presencia obligada de los participantes al momento de negociar, promoviendo mayor inversión de tiempo y dinero en el proceso en general. Nuestra propuesta además de abarcar los problemas antes mencionados intenta también fomentar una comunicación más efectiva entre los miembros participantes, explorar los aspectos involucrados en el proceso de negociación de una manera creativa, efectiva y finalmente construir un entendimiento compartido.

Teniendo en cuenta que uno de los puntos centrales para facilitar el trabajo de equipos móviles es la creación y establecimiento de equipos desde agentes autónomos. Nuestro propósito es aplicar una nueva metodología para proveer mecanismos que faciliten la creación de equipos y ayudar a resolver conflictos a través de negociación automatizada. Los mecanismos de negociación serán implementados mediante una combinación de la teoría de juegos y la metodología co-evolutiva.

Este artículo está organizado así: En la sección 2 se hace una breve introducción sobre negociación y de igual forma se toca la negociación automatizada. La

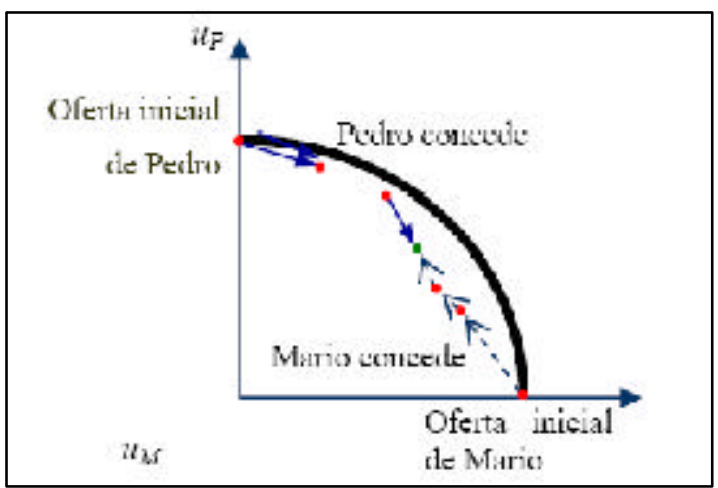

Figura 2. Esquema de negociación entre dos participantes, hay ofertas, concesiones hasta que se da el acuerdo.

sección 3 trata sobre el enfoque de la teoría de juegos, algunos conceptos que se deben tener claros y los avances que a permitido este enfoque a la negociación. La sección 4 ofrece un resumen acerca del tratamiento que a dado el enfoque co-evolutivo a la negociación. La sección 5 presenta el mecanismo de negociación automatizada que se desarrollará. En laúltima sección presentamos algunas conclusiones y el dominio real de aplicación.

SOBRE

NEGOCIACIÓN

Los distintos métodos que ayudan a la negociación, enmarcan lo que podemos llamar una "tecnología para la cooperación" [16] que halla sus raíces en el análisis de decisiones y la teoría de juegos misma, dentro de la negociación, los medios que conllevan a un estado cooperante son la presentación de propuestas, opciones de comercio, realización de concesiones y (es lo que se espera) un acuerdo aceptado mutuamente, ver Figura 2.

Una perspectiva útil en negociación es ver esta como una búsqueda distribuida en un espacio de potenciales acuerdos, la dimensionalidad y topología de dicho espacio depende de la estructura de los objetos que entran en la negociación [10]. Muchas definiciones de negociación han sido ofrecidas, por diferentes investigadores, dependiendo de los propósitos de su investigación ([15], [21]). Por ejemplo:

- [9] define negociación como un tipo de proceso de resolución de problemas, en el cual las personas intentan encontrar una decisión conjunta sobre temas de interés común en situaciones donde ellos están en desacuerdo y en conflicto.

- En [15] definen negociación como una forma de proceso de toma de decisión donde dos o más 
partes conjuntamente buscan en un espacio de posible solución con el objetivo de encontrar un consenso (trato).

Una diferencia principal entre estas definiciones es que el antiguo énfasis se centró en resolver conflictos y desacuerdos, mientras el posterior pensamiento sobre negociación es que puede ser una metodología para mejorar la cooperación. No necesariamente existe conflicto entre los participantes de la negociación [15].

La ineficiencia ha sido ampliamente reconocida como un problema común en negociación [11], muchos proyectos de investigación ([1], [5]) han sido conducidos para mejorar la eficiencia en negociación usando tecnología computacional.

\section{Negociación Automatizada}

Un número creciente de sistemas computacionales están siendo abarcados en términos de agentes autónomos, hay dos principales razones que conducen a esto: La primera es que los agentes están siendo abocados como la próxima generación de modelos para sistemas complejos, la segunda que estos son usados dentro de un esquema de trabajo el cual une distintas sub-disciplinas de la Inteligencia Artificial que son necesarias para diseñar y construir entidades inteligentes.

Cuando se adopta una visión de la computación orientada-a-agentes, se nota como en la mayoría de problemas se requiere involucrar múltiples agentes que representen la naturaleza descentralizada del problema. La tecnología de sistema multi-agente (MAS) da una oportunidad para superar el problema de ineficiencia en negociación, es decir, nos brinda la posibilidad de manejar las múltiples variables involucradas, las interacciones complejas y el inadecuado conocimiento de negociación de los participantes. En este sentido, los agentes en MAS actúan colectivamente como una sociedad y ellos colaboran (o cooperan) para lograr sus propios objetivos individuales así como también los objetivos globales de la sociedad a la cual ellos pertenecen [13].

Teniendo en cuenta el MAS como una solución a la ineficiencia en negociación, otras investigaciones dieron paso a la llamada "negociación automatizada" la cual puede ser considerada al tratar con tres grandes asuntos [10]:

- Protocolos de negociación: el conjunto de reglas que gobiernan la interacción.

- Objetos de negociación: el rango de asuntos sobre los cuales el acuerdo debe ser logrado.

- El modelo de toma de decisión de los participan- tes: el mecanismo de toma de decisión que los participantes emplean al actuar de acuerdo con el protocolo de negociación para lograr sus objetivos.

Además, teniendo en cuenta la definición sobre negociación en [15], en la comunidad de inteligencia artificial distribuida (DAI) se gestaron algunos proyectos de investigación en los cuales, "el principal asunto en la investigación de sistemas multi-agentes es como modelar las interacciones entre los agentes. El modelo de negociación ha surgido como un candidato apropiado para resolver este problema de interacción debido a su naturaleza descentralizada, énfasis sobre selección mutua de una acción y la prevalencia de la negociación en sistemas sociales reales" [15].

Incluso muchas teorías importantes y principios han sido desarrollados para explicar diferentes aspectos de negociación, tales como la metodología organizacional, la metodología estructural, la metodología de la teoría de juegos, la metodología de la teoría económica, la metodología de la teoría del comportamiento y la metodología de experimentación y simulación.

ENFOQUE DE LA TEORÍA DE JUEGOS

La teoría de juegos nació con el objetivo de analizar el comportamiento de las personas en situaciones con conflictos de intereses, es decir, nos ayuda a crear modelos sobre los cuales razonamos para elegir las estrategias que nos conduzcan a resultados óptimos, donde todos los participantes del proceso ganen.

Gracias a la teoría de juegos se han realizado muchas investigaciones sobre negociación automatizada, tratando siempre de obtener mejores resultados del proceso de negociación. Gracias a estos estudios contamos con conceptos como:

Estrategias dominantes Una estrategia dominante para un jugador es una estrategia que siempre le dará una retribución más alta sin importar las estrategias que los otros jugadores escojan.

Equilibrio de Nash

Una combinación estratégica $\left(S_{i}{ }^{*}, S_{-i}{ }^{*}\right)$ es un equilibrio de Nash si cualquier agente que se desvíe de forma unilateral obtendrá menos retribución. Matemáticamente:

$$
\forall i, \pi_{i}\left(s_{i}^{*}, s_{-i}^{*}\right)>\pi_{i}\left(s_{i}^{\prime}, s_{-i}^{*}\right), \forall s_{i}^{\prime}
$$


Eficiencia de Pareto

Una combinación estratégica es una eficiencia de Pareto si no hay otra combinación estratégica que incremente la retribución de un agente sin decrementar la retribución de otro agente.

\section{Forma extensiva}

Es la forma de representar un juego por medio de un grafo (un conjunto de aristas y nodos) también conocido como árbol del juego, en donde los nodos representan a los participantes del juego y las aristas las estrategias de dichos participantes.

\section{Forma normal}

La forma normal de un juego es una lista de todas las estrategias para todos lo jugadores, junto con las retribuciones esperadas para cada posible elección de estrategias (una para cada agente).

El dilema del prisionero

Veamos el siguiente caso. Dos prisioneros $A$ y $B$ se encuentran encerrados en celdas separadas acusados de algún delito. Ambos van a ser interrogados por separado por sus carceleros. A cada uno se le preguntará por la culpabilidad del otro. Cada preso puede optar por "Colaborar" con el otro, asegurando que el compañero se encuentra injustificadamente en la cárcel, o "Defraudar" acusándole. Existen por tanto cuatro posibilidades, que ninguno defraude, que lo hagan los dos, que lo haga el primero o el segundo [2].

Cada prisionero recibe un premio en función del Cuadro 1, mayor cuanto mayor es el número que aparece a la derecha. El -1 es una pena de cárcel de 10 años, el 0 es una pena de cárcel de 5 años, el 3 la libertad; y el 5 la libertad y una indemnización.

Supongamos que somos el jugador B. Si el jugador A defrauda (Confiesa) es indiferente lo que hagamos, ya que recibiremos el castigo. Pero si el jugador $A$ coopera con nosotros (no confiesa), el premio recibido es mayor si nosotros a su vez le defraudamos a él. En resumen, haga lo que haga el jugador $A$, para el jugador $B$ lo mejor es defraudar (confesar) y lo mismo ocurre para el jugador $A$ : haga lo que haga el jugador $\mathrm{B}$, lo mejor es defraudar (confesar).

Cuadro 1. Matriz de pagos en el Dilema del Prisionero

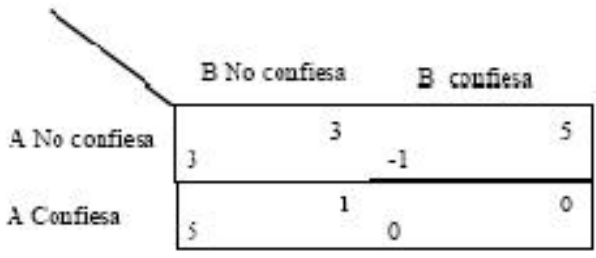

Ya que las decisiones son independientes, y dado que el objetivo de cada uno es lograr el máximo beneficio personal, lo racional es defraudar. Pero si los dos se comportan racionalmente, ambos recibirán el castigo [17].

Avances en la negociación mediada por ju g gos

Los agentes racionales son capaces de llegar a acuerdos con un mecanismo de negociación de la teoría de juegos aún sin comunicación, esto ocurre cuando los agentes de forma aislada escogen el punto que ellos consideran es el equilibrio [2], [8]. Sin embargo, surgen problemas con los mecanismos de negociación en la teoría de juegos sin comunicación, el primero es que en situaciones parecidas a la del dilema del prisionero los agentes no escogerán el mejor resultado basados en la suposición de racionalidad [8], [12]. En segundo lugar, aquellos juegos en los cuales no existen puntos de equilibrios, encontraremos ciclos infinitos de razonamiento, aunque estos juegos utilizan el concepto de estrategias mixtas para solucionarse esta no es la mejor opción [4], [18]. En tercer lugar cuando encontramos más de un equilibrio es difícil seleccionar el mejor, ya que cada agente puede tender a puntos diferentes según sus objetivos particulares [18].

En [19], se propuso la primera acción de comunicación dentro de la convencional teoría de juegos, pedir garantía, para escapar de situaciones como la del dilema del prisionero. Para los casos en donde no existen equilibrios o hay múltiples equilibrios se propuso la segunda acción de comunicación, pagar compensación. Además, estas acciones de comunicación exigen la existencia de un agente independiente del dominio de negociación, denominado mediadoro tercera parte confiable [18].

Hasta aquí todo el trabajo que se había realizado era en juegos con información completa, es decir, además de conocer sus retribuciones al momento de combinar sus estrategias con las estrategias de los otros agentes, también conocían las retribuciones de los otros agentes, pero esto no es lo ideal ya que no refleja la realidad.

Luego en [20] se trataron juegos con información incompleta (cada agente solo tiene conocimiento de sus propias retribuciones y no de las retribuciones de lo demás agentes) siguiendo la misma filosofía de las acciones de comunicación de garantía y compensación, las cuales convertían un juego difícil (ya que un juego puede no tener o tener muchos puntos de equilibrios en estrategias puras, este tipo de juegos surgieron como juegos difíciles) en un juego simple. 
La acción de pedir garantía consiste, en que un agente le solicite a otro agente que le pague cierta garantía de no jugar alguna estrategia que conduzca a un estado no deseable, por otro lado la acción de pagar compensación se utiliza para convencer a otro agente de que juegue una estrategia particular a cambio de una recompensa. El equilibrio alcanzado por medio de las acciones de comunicación se denomina equilibrio NFD (sin temor de desviación), el cual equivale a un equilibrio de Nash y también a una eficiencia de Pareto.

ENFOQUE

CO-EVOLUTIVO

Los campos de la economía y ciencias de la administración han sido los primeros en la aplicación de enfoques evolutivos para la resolución de conflictos ([2], [13]). El caso del juego iterado proporcionó un marco de trabajo en la investigación del refinamiento de equilibrios de Nash, y de su aclaración como equilibrio aprendido ([7]). Aunque el enfoque evolutivo para la negociación automatizada, proporcione una alternativa al enfoque de teoría de juegos en la resolución del conflicto, este sufre algunas limitaciones. El enfoque Co-evolutivo asume la existencia de un equilibrio de Nash que puede ser refinado, en razón a queen estas técnicas hayan soluciones aproximadas, a menudo el enfoque co-evolutivos produce soluciones sub-óptimas.

A continuación se esboza el trabajo presentado en [3]. El punto inicial de la metodología co-evolutiva para la negociación es la disponibilidad de un conjunto de estrategias iniciales, las cuales son posteriormente manipuladas para encontrar una solución optimizada de un problema específico. Esto involucra el desarrollo de una función de utilidad para evaluar estrategias, así cada estrategia tendrá asignada un valor único. La función de utilidad usará este valor para determinar el grado de su utilidad como una solución. La generación de una estrategia optimizada o solución involucra la selección de la mejor solución, incluyendo los padres y la creación de descendientes a través de la mutación y el cruzamiento. La próxima generación es seleccionada por medio de un proceso de competencia o torneo. Las soluciones más débiles son removidas desde la población y reemplazadas por las mejores soluciones. Un sistema co-evolutivo será implementado para soportar negociación entre múltiples agentes que puedan manejar varios asuntos. Bajo este esquema, el sistema provee una estrategia general desde la que genera de forma aleatoria un número de estrategias específicas que construirán la población inicial. Cada estrategia es codificada dentro de su correspondiente cromosoma.
Un agente está en capacidad de especificar su propia función de utilidad. Las estrategias pueden ser refinadas por medio de la función de utilidad, las cuales pueden ser ajustadas cambiando sus parámetros. La más alejada del umbral es la estrategia adecuada.

Además, la tasa de mutación y la tasa de cruzamiento pueden ser determinadas arbitrariamente por el agente. El proceso de negociación es iniciado por un agente, y sostenido por intercambio de ofertas y contraofertas las cuales constituyen los fundamentos del protocolo. Aunque convergen hacia una solución común es deseable y aceptable no representar una solución optimizada global. Esto es la realización que atribuye la incorporación de aceptación o rechazo de ofertas dentro del protocolo. Sin embargo, esto permite a un agente hacer una oferta que fue peor que una previa. Estas características permiten al sistema buscar en todo el espacio de potenciales acuerdos. Los agentes negocian sobre un número de asuntos y las estrategias son creadas y seleccionadas en con el propósito de maximizar su ganancia con respecto a estos asuntos.

MECANISMO DE NEGOCIACIÓN

Basándonos en los enfoques propuestos en [3] y [20], se presenta ahora de forma general el mecanismo de negociación que se propone usar, conllevando un proceso que soporta la negociación automatizada en la cual se obtiene por resultado un equilibrio con altas retribuciones, que a su vez es equivalente a un equilibrio de Nash y una eficiencia de Pareto. Dicho proceso les permite a los agentes generar y seleccionar estrategias eficientes bajo las cuales se negocia.

El sistema de negociación automatizada está compuesto por dos clases de agentes: Agente Negocia-

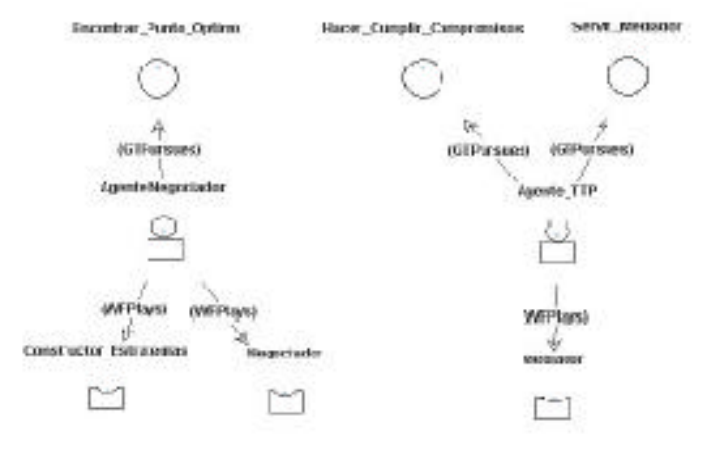

Figura 3. Modelo de Agentes 
dor y Agente TTP. El Agente Negociador, es el que representa al usuario en el proceso de negociación automatizada del sistema, además de negociar, su objetivo es lograr un acuerdo con el mayor grado de utilidad posible. El Agente TTP, es el encargado de conducir el proceso de negociación automatizada y en ese sentido hace llegar las propuestas entre los agentes negociadores, ejecuta las acciones de comunicación (garantía y/o compensación) y hace cumplir los compromisos pactados, ver Figura 3.

El protocolo de negociación y la estrategia son dos importantes constituyentes en el mecanismo utilizado, los cuales suministran a los agentes negociadores las reglas de interacciones y directivas para la comunicación durante la negociación. Ellos también determinan cuándo el proceso de negociación termina.

Básicamente el sistema de negociación propuesto funcionará así: El administrador del sistema ingresará información relevante para la ejecución del software, por ejemplo: el número de agentes negociadores, las etapas de negociación que se llevarán a cabo y el numero de iteraciones que se ejecutarán en cada etapa, etc. Posteriormente cada uno de los Usuarios del Sistema al interactuar con el soft- ware, personaliza al agente que lo representará en la negociación.

Cada agente negociador en el sistema incorpora dos componentes principales: un Algoritmo Genético y un Algoritmo de Equilibrio NFD. Los dos componentes intercambian información internamente por medio de una matriz de retribuciones, generada por la metodología co-evolutiva. Cada agente negociador implementa un ciclo sobre el cual genera y selecciona estrategias, codifica la matriz de retribuciones y determina un punto optimizado. El punto óptimo puede ser usado como un punto de referencia para que los agentes negociadores exploren más el espacio de posibles acuerdos. Además, los agentes negociadores intercambian información externamente por medio de un protocolo.

Los agentes deben negociar sobre los mismos asuntos y cuando un agente hace una oferta, el otro agente debe hacer una contra oferta, aceptarla o rechazarla.

El proceso de negociación finalmente terminará su ejecución cuando se cumpla el número de etapas de negociación, en lo posible, con un acuerdo; posteriormente se mostrará el resultado del proceso de

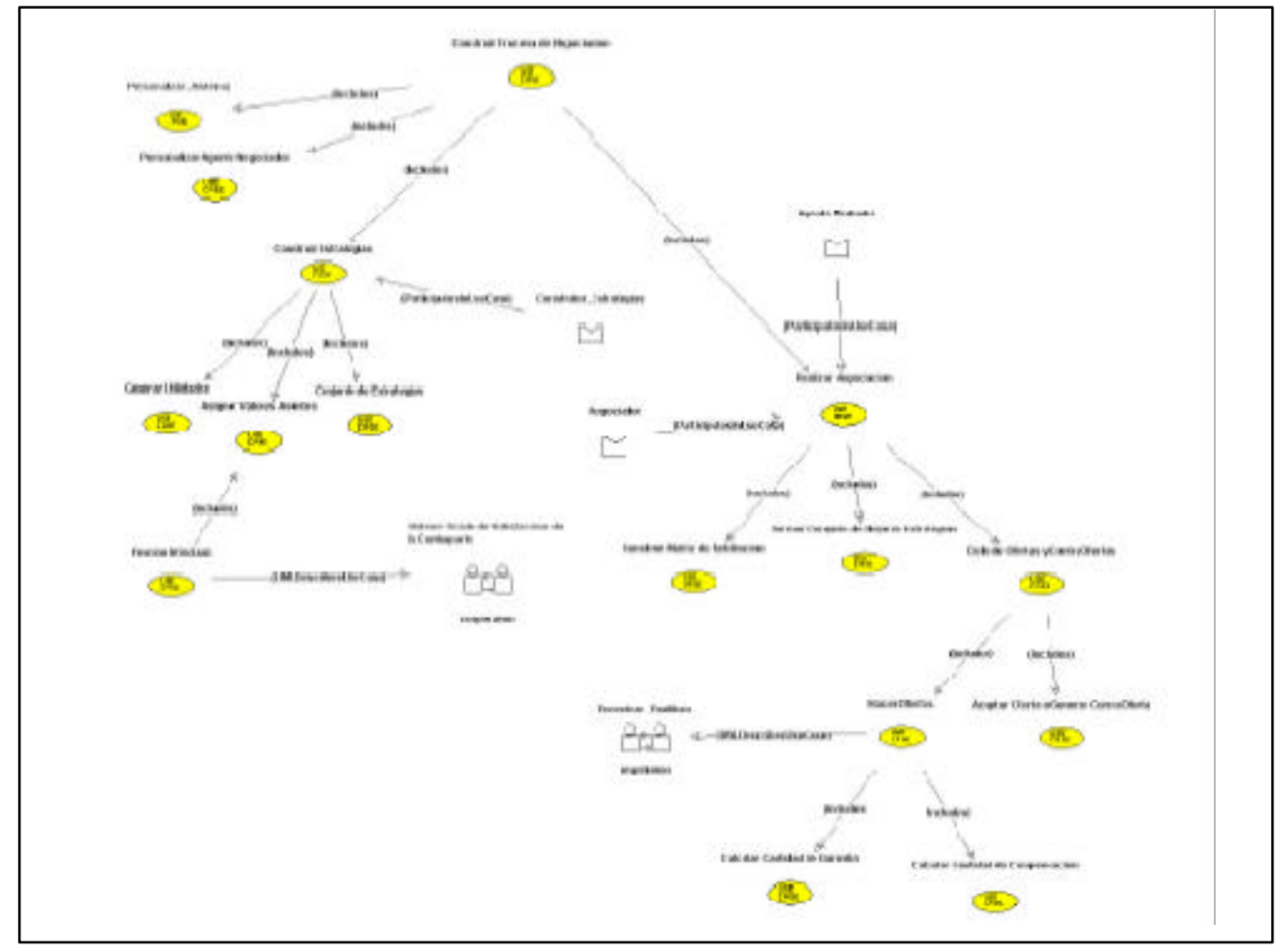

Figura 4. Funcionamiento General del Sistema de Negociación Automatizada 
negociación automatizada a todos los usuarios del sistema, ver Figura 4.

De igual forma, el sistema de negociación posee las siguientes características:

- Los agentes solamente tienen acceso cada uno a su matriz de retribuciones y función de utilidad, no tienen conocimiento de las retribuciones y función de utilidad de los otros agentes.

- Ya que el proceso co-evolutivo es responsable de encontrar altas retribuciones para los agentes negociadores buscando en el espacio de potenciales acuerdo, el trato o acuerdo no puede ser hecho en este nivel. Esto asegura que el espacio de potenciales acuerdos es explorado completamente y los agentes actúan de una forma racional, por ejemplo, no aceptar una oferta que es menor a una anterior.

- El algoritmo de equilibrio NFD es aplicado a la matriz de retribución, cuando es requerido, obtener un equilibrio de Nash que también es eficiencia de Pareto.

- Si el equilibrio NFD no satisface los requerimientos de los agentes, la fase co-evolutiva es revisada para seleccionar una nueva estrategia.

- Una vez los agentes acuerdan sobre las retribuciones producidas por el algoritmo NFD, es necesario determinar las estrategias que conducen a estas retribuciones. Para este fin el proceso del GA es invocado con la función de adaptabilidad ajustada para reflejar el nuevo equilibrio NFD. Como el GA no puede ser capaz de encontrar una correspondencia exacta a la retribución asociada con el equilibrio NFD, una solución aproximada es aceptable.

- Las acciones de comunicación de Compensación y Garantía son incorporadas para permitirle a los agentes reevaluar las retribuciones en la matriz en su búsqueda de un equilibrio.

- El sistema se detiene cuando un acuerdo mutuamente aceptable es logrado siguiendo un nuevo equilibrio o cuando los recursos de los agentes se hayan agotado.

C O N C L U S I O N E S

El trabajo presentado es un análisis sobre la negociación automatizada, desde dos perspectivas fundamentales, teoría de juegos y la metodología co- evolutiva. El primer tema que se tiene en cuenta desde la teoría de juegos es el equilibrio de Nash. Aunque una solución de negociación acorde a Nash ofrece una interesante y útil perspectiva sobre el comportamiento de la negociación, falla para negociar con juegos donde se traten múltiples asuntos o se utilicen estrategias con información incompleta. La teoría de juegos puede tratar múltiples temas, pero dentro de un espacio de búsqueda limitado y con información completa. La adopción de una metodología co-evolutiva para negociación automatizada permite a los usuarios ignorar mucha de las suposiciones requeridas por la metodología de teoría de juegos.

La condición requerida por las metodologías de AG y teoría de juegos cuando ellas operan aisladamente restringe su rango de aplicación. El sistema de negociación ofrece una simbiosis entre una metodología de AG y una metodología de teoría de juegos que supera sus limitaciones inherentes. La suposición de la teoría de juegos de la existencia de una matriz de retribución con el propósito de alcanzar un equilibrio de Nash es frecuentemente difícil de cumplir. La metodología de AG es, por otra parte, capaz de generar un conjunto de estrategias efectivas y relacionar retribuciones para proveerlas a la metodología de teoría de juegos. La metodología de AG requiere, sin embargo, la presencia de un equilibrio de Nash. En cambio, la metodología de teoría de juegos puede determinar un equilibrio sin temor a desviación desde la matriz de retribución y así poder proveer un punto focal para la metodología de AG, la cual permite refinar el equilibrio. Algunas de las ventajas que conlleva esta combinación es que es posible para los agentes negociantes llegar a un acuerdo sin conocer las retribuciones de cada uno de los agentes participantes en la negociación. La transparencia ofrecida por el sistema facilita el establecimiento de conexiones y la creación de equipos.

Los modelos presentados en este artículo fueron generados gracias a la metodología Ingenias, teniendo en cuenta sus fases de Análisis y Desarrollo [24].

El dominio real de aplicación en el que se piensa implementar nuestra propuesta es el de elaboración de presupuestos en la Jefatura Financiera de la Vicerectoría Administrativa y Financiera de la Universidad del Magdalena.

REFERENCIAS

BIBLIOGRÁFICAS

1. Anson, R. and T. Jelassi. (1990). A Development Dramework for Computer-supported Conflict Resolution. EJOR, 46, 181-199. 
2. Axelrod, R. (1984). The Evolution of Cooperation, Basic Books Inc., New York, USA.

3. Chen, J-H., Anane, R., Chao, K-M., Godwin, N. (2002). Architecture of an Agent-Based Negotiation Mechanism. Proceedings of the 22nd IEEE International Conference on Distributed Computing Systems Workshop (ICDCS-2002), Vienna, July 2002, 379-384.

4. Durfee, E.H., Lee, J., Gmytrasiewicz, P.J. (1993). Overeager Reciprocal Rationality and Mixed Strategy Equilibria, In Proceedings of the Eleventh National Conference on Artificial Intelligence (AAAI93), 225-230.

5. Ephrati, E., Rosenschein, J.S. (1996). Deriving Consensus in Multi-agent Systems. Journal of Artificial Intelligence. Volume 87, Numbers 1-2, 21 74.

6. Faratin, P. (2005). Automated Service Negotiation Between Autonomous Computational Agents. A dissertation submitted in partial fulfilment of the requirements for the degree of Doctor of Philosophy of the University of London.

7. Fudenberg D., Levine D.K. (1998). The Theory of Learning in Games, Cambridge: M.I.T. Press.

8. Genesereth, M.R., Ginsberg, M.L.,. Rosenschein, J.S. (1986). Cooperation without communication, In Proceedings of the National Conference on Artificial Intelligence (AAAI-86), Philadelphia, Pennsylvania, 51-57.

9. Gulliver, P.H. (1979). Dispute and Negotiations: A Cross Cultural Perspective. Academic Press, New York, USA.

10. Jennings, N. R., Faratin, P., Lomuscio, A. R, Parsons, S., Sierra, C. and Wooldridge, M. (2001). Automated negotiation: prospects, methods and challenges. Int. J. of Group Decision and Negotiation 10 (2) 199-215.

11. Kraus., S.: An Overview of Incentive Contracting (1996). Artificial Intelligence journal, 83 (2) 297346.

12. Mor, Y., Rosenschein, J. S. (1995). Time and the Prisoner's Dilemma, In Proceeding of the First International Conference of Multi-Agent System (ICMAS-95), 276-282.

13. Ren, Z., Anumba, C. J., Ugwu, O. O. (2005). Negotiation in a multi-agent system for construction claims negotiation; Centre for
Innovative Construction Engineering (CICE). Department of Civil \& Building Engineering Loughborough University, Loughborough, UK.

14. Ríos Insua, D., Fernández, E., Ríos, J. (2004). Más allá del gobierno electrónico: hacia la democracia electrónica. Revista de la Agencia de Protección de Datos de la Comunidad de Madrid.

15. Rosenschein, J. S., Zlotkin, G. (1994). Rules of Encounter: Designing Conventions for Automated Negotiation Among Computers. MIT Press, Cambridge, Massachusetts, USA.

16. Sebenius, J. K. (1998). Negotiation Analysis: A Characterization and Review. Management Science 38(1) 18-38.

17. Von Neumann, J. Morgerstern, O. (1944). The Theory of Games and Economic Behaviour. Princeton University Press.

18. Wu, S.H., Soo, V.W. (1999). Game Theoretic Reasoning in Multi-agent Coordination by Negotiation with a Trusted Third Party, in Proceedings of the Third International Conference on Autonomous agents (Agents'99), Seattle, Washington, 56-61.

19. Wu, S.H., Soo, V.W. (1998). Escape from a Prisoners' Dilemma by Communication with a Trusted Third Party, ICTAI-98.

20. Wu, S.H., Soo, V.W. (200). Negotiation Without Knowing Other Agents Payoffs in the Trusted Third-Party Mediated-Game, Second workshop on game theoretic and decision theoretic agents.

21. Zartman, I.W., BermanN, M. (1982). The Practical Negotiator. Yale University Press, New Haven, Conn.

22. Souza, C. U.: Participatory budgeting in brazilian cities: limits and possibilities in building democratic institutions. Environment and Urbanization, 131 (2001)159-184.

23. J. Rios, D. Rios Insua, E. Fernandez, and J.A. Rivero. (2005). Participatory Budget Formation Through the Web. TCGOV 2005, LNAI 3416, pp. 268-276.

24. Grasia! Research Group (2004). INGENIAS Development Kit:Tutorial and Manual. Technical Report. Facultad de Informática, Universidad Complutense de Madrid, Spain.Available at http:/ /ingenias.sourceforge.net. 